Revue d'histoire du XIXe siècle

Société d'histoire de la révolution de 1848 et des

révolutions du XIXe siècle

$52 \mid 2016$

Chrononymes. Dénommer le siècle

\title{
« Printemps des peuples » : pour une autre lecture des révolutions de 1848
}

The "Printemps des peuples" (Springtime of the peoples). For another understanding of the 1848 revolutions

Der Völkerfrühling : für eine andere Lesart der Revolutionen von 1848

\section{Jean-Claude Caron}

\section{OpenEdition \\ Journals}

Édition électronique

URL : http://journals.openedition.org/rh19/4988

DOI : $10.4000 /$ rh 19.4988

ISSN : $1777-5329$

Éditeur

La Société de 1848

Édition imprimée

Date de publication : 1 juin 2016

Pagination : $31-45$

ISSN : 1265-1354

Référence électronique

Jean-Claude Caron, « «Printemps des peuples » : pour une autre lecture des révolutions de 1848 », Revue d'histoire du XIXe siècle [En ligne], 52 | 2016, mis en ligne le 01 juin 2019, consulté le 10 décembre 2020. URL : http://journals.openedition.org/rh19/4988 ; DOI : https://doi.org/10.4000/ rh19.4988 


\section{JEAN-CLAUDE CARON \\ "Printemps des peuples»: \\ pour une autre lecture des révolutions de 1848}

L'expression "Printemps des peuples», qui associe un acteur collectif à une saison, désigne un mouvement révolutionnaire européen se déroulant pour l'essentiel entre fin février et début juillet 1848. Eric J. Hobsbawm l'interprète comme «the first and last European revolution in the (almost) literal sense», fondant son propos sur la simultanéité du déclenchement des insurrections et sur la dilatation de l'espace concerné, «de Copenhague à Palerme, de Brasov à Barcelone " ${ }^{1}$. Si les foyers révolutionnaires se multiplient alors (Paris, Berlin, Munich, Vienne, Milan, Turin, Venise, Rome, Budapest, Prague, Bucarest), il subsiste toutefois, pour des raisons diverses, des zones blanches (Saint-Pétersbourg, Londres, Madrid, Bruxelles). La forte concentration d'événements entre mars et juin 1848 est censée donner à cette séquence sa dimension saisonnière, en l'occurrence printanière. Mais ce balisage temporel est rien moins que neutre car il ne rend pas compte de la dilatation spatio-temporelle de l'événement «Printemps des peuples». D'une part, la séquence inaugurale de ce mouvement révolutionnaire peut aussi bien être associée aux barricades érigées à Palerme ou à Naples en janvier 1848, intégrer la fin du Vormärz allemand marquée par d'intenses tensions sociopolitiques dans la Confédération germanique, prendre en considération la guerre du Sonderbund qui touche la Suisse en novembre 1847, l'insurrection qui éclate à Cracovie en février 1846, comme y invite François Fejtö dans Le Printemps des peuples ${ }^{2}$, ou encore l'élection de Pie Ix à Rome, qui inaugure des réformes libérales dans les États pontificaux, mais aussi en Toscane et au Piémont. D'autre part, le "Printemps des peuples» se poursuit dans certains cas jusqu'à l'automne 1848 (à Vienne), voire jusqu'à l'été 1849 (en Hongrie, au Piémont, à Venise, à Rome). François Fejtö propose la date terminale de décembre 1851, justifiée par le coup d'État de Louis Napoléon Bonaparte en France et par la suppression de la constitution en Autriche ${ }^{3}$. On peut donc à

1. Eric J. Hobsbawm, L’Ėre du Capital, 1848-1875, Paris, Fayard, 1977 [1975].

2. Voir le «Tableau chronologique» qui ouvre l'ouvrage publié en 1948 aux Éditions de Minuit, tome I, p. 11.

3. Ibidem, p. 24 
bon droit plaider pour une définition extensive d'un printemps révolutionnaire quinquennal ${ }^{4}$. Quant à l'interprétation de ses causes et de ses objectifs, sa répression par des souverains absolutistes comme le tsar Nicolas I ${ }^{\text {er }}$, l'empereur François-Joseph ou le roi Frédéric-Guillaume IV, renforce le sentiment que ces mouvements étaient majoritairement libéraux et nationaux, quitte à occulter la question de la souveraineté populaire ou la question sociale qui se posèrent alors avec acuité. En utilisant l'expression "Printemps des Peuples", on écarte toute filiation directe des révolutions de 1848 avec l'émergence de revendications sociales qui virent le jour dans de nombreuses régions touchées par la crise économique qui frappa durement l'Europe à partir de 1846. On peut également évoquer la mémoire des expériences insurrectionnelles et révolutionnaires des années 1820 et 1830, que résume incomplètement le mot de Charbonnerie. Ce faisant, il ne s'agit ni de proposer une dilatation temporelle hors de propos du "Printemps des peuples", ni de plaider pour une sorte de continuum révolutionnaire, mais d'affirmer que la séquence révolutionnaire européenne du printemps 1848 s’inscrit dans une généalogie et ne peut être comprise qu'en variant les échelles de temps et d'espace.

\section{UN PLURIEL SIGNIFICATIF}

Comme d'autres chrononymes, "Printemps des peuples» est révélateur de la «bataille de mots» qui s'empare de l'événement, le façonne pour lui donner un sens unique, généralement celui des détenteurs de pouvoir. La comparaison avec les "Trois Glorieuses» est éclairante. Après un temps de fluctuation identitaire, où la révolution de Juillet est plus ou moins dilatée, l'orthodoxie chronologique s'impose par la volonté des vainqueurs. Soucieux de limiter la durée d'une séquence que d'aucuns se refusent à qualifier de révolutionnaire, ils mobilisent la mémoire de la Glorieuse Révolution anglaise, modèle de changement de régime non-violent, à la durée et à la portée limitées. L'expression "Trois Glorieuses", à laquelle on accole parfois le mot de «journées", permet d'euphémiser la dimension révolutionnaire de l'événement. Là s'arrête la comparaison. Car, alors que les expressions "Trois Glorieuses" ou "Semaine sanglante» ont été produites à chaud, tel n'est pas le cas du «Printemps des Peuples», du moins dans l'espace français. Il convient donc de retracer la généalogie de ce chrononyme, dont on verra qu'il est largement partagé par les historiographies européennes de l'année 1848 - mais pas forcément dans le même sens. On interrogera la pluralité de cet acteur collectif, les "peuples", au regard d'un emploi très germano-centré du chrononyme. D'autant qu'on peut parler de réemploi, comme, du reste,

4. Jacqueline Lalouette définit ainsi le «Printemps des peuples» comme "l'ensemble des mouvements révolutionnaires qui traversèrent la quasi-totalité de l'Europe de 1847 à 1849" (Les Mots de 1848, Toulouse, Presses Universitaires du Mirail, 2007, p. 101). 
de réactualisation de la formule à trois époques au moins : les lendemains de la Seconde Guerre mondiale et le début de la guerre froide, la partition de l'Europe en deux blocs coïncidant avec le centenaire du "Printemps des peuples "; la chute des régimes communistes d'Europe de l'Est entre la fin des années 1980 et le début des années 1990 ; et le "printemps arabe» qui débute fin 2010, une appellation qui a déclenché un vif débat en France.

La fortune de l'expression "Printemps des peuples» est visible à l'aune des nombreuses traductions - et parfois adaptations - dont elle a été l'objet dans les différentes langues européennes. "Primavera dei popoli» fait écho à «Primavera de los Pueblos»; «Springtime of peoples» à "Völkerfrühling»; "Весна народов» (russe) à "Jaro národů " (tchèque); "Wiosna Ludów" (polonais) à «Proljeće naroda» (croate); "Jar národov» (slovaque) à «népek tavasza" (hongrois) 5 . Ces expressions demeurent parfois soumises à concurrence : en Espagne, pour désigner 1848, on évoque aussi bien l'"Año de las Revoluciones»; en Angleterre, on parle également de "Springtime of Nations»; et en Allemagne, 1848 fut très vite surnommé, par les vainqueurs du Printemps des peuples, "das tolle Jahr», c'est-à-dire l'année folle. Il reste par ailleurs peu de traces de cette séquence révolutionnaire dans le langage, sauf dans la langue italienne où les expressions "fare un quarantotto", "combinare un quarantotto" ou "essere un quarantotto" désignent une situation désordonnée, chaotique, voire sanglante.

Peuples, popoli, pueblos, peoples, Völker: l'acteur de ce printemps est moins un acteur collectif singulier - «le peuple» - qu'une pluralité d'acteurs nationaux - «les peuples». Le mot allemand Volk/Völker traduit cette dimension nationale du peuple, fondée sur une histoire et une culture communes s'appuyant sur une mythologie des origines. D'une certaine manière, «Printemps des peuples" s'inscrit dans la continuité de la sémantique développée dans l'Europe des Restaurations. Les "libéraux» des différentes nations y proclamèrent la lutte entre «la Sainte-Alliance des rois» et «la Sainte-Alliance des peuples» - on sait que Béranger popularisa la chose dans l'une de ses plus célèbres chansons ${ }^{6}$. On est donc loin de la conception sociale et encore plus classiste $d u$ peuple telle que les réformateurs sociaux la développèrent jusqu'à «l'ouvriériser», puis la "prolétariser» à l'image de Blanqui ou de Marx. Ce qui, du reste, n'empêcha nullement ce dernier de célébrer le chansonnier français dans une lettre adressée au Gouvernement provisoire français le 28 février 1848 : «À vous Français, à vous l'honneur, à vous la gloire d'avoir jeté les fondements de cette alliance des peuples si prophétiquement chantée par votre immortel Béranger ${ }^{7}$.

5. Je remercie Marie-Pierre Rey et Antoine Mares qui m’ont fait bénéficier de leurs compétences linguistiques et Landry Charrier de ses compétences bibliographiques.

6. Pierre Jean de Béranger, "La Sainte Alliance des Peuples» [1818], Euvres complètes, Paris, H. Fournier, 1839, tome 2, p. 26-28.

7. Cité par Dietmar Rieger [dir.], La Chanson française et son histoire, Tübingen, Gunter Narr Verlag, 1988, p. 107. 
Cette alliance ne peut pour autant être conçue comme une forme de proto-internationalisme : ainsi qu'en témoigne l'iconographie du printemps 1848, les peuples convoqués pour illustrer la concomitance de leur action révolutionnaire défilent avec les drapeaux de leur pays - ou dont ils souhaiteraient qu'ils le deviennent, comme les drapeaux tricolores allemand et italien ${ }^{8}$. Par ailleurs, la pertinence même des notions de "peuple allemand" ou de "peuple italien" mérite d'être interrogée, tant les identités régionales demeurent fortes. Dans le cas de l'espace germanique, l'usage du terme "allemand" est dès l'origine éminemment politique, comme en témoigne la «bataille des nations» (Leipzig, 16-19 octobre 1813), qualifiée de Völkerschlacht dans l'historiographie allemande qui a construit l'événement comme le fondement de l'unité nationale. En témoigne notamment la construction d'un Völkerschlachtdenkmal inauguré en octobre 1913 par l'empereur Guillaume II. Et l'on sait la rivalité séculaire qui opposa la Prusse et l'Autriche pour patronner une germanité culturelle, voire politique, mais dont la traduction étatique se heurta à des projets divergents, ce que confirma avec brutalité le «Printemps des peuples».

\section{UN PRINTEMPS BIEN PEU FRANÇAIS}

La quête de l'invention d'un mot ou d'une expression est toujours soumise au risque de trouver des antécédents. D'autant que cette expression peut changer de signification. "Printemps des peuples» est par exemple utilisé en 1790 dans une vibrante apologie des idéaux de la Révolution française, rappelant que : «L'ignorance et la simplicité sont le printemps des peuples; les sciences, les arts et la philosophie en sont l'été»". En l'absence de sources attestant la diffusion de la formule dans d'autres publications contemporaines ou postérieures, on conclura que la métaphore poétique ainsi produite n'a pas été investie et n'a pas, alors, revêtu de dimension politique. De fait, les sources françaises des décennies suivantes ne permettent pas de repérer la réitération de la formule avant l'année 1848. Non que la référence européenne soit absente du discours politique de la période, en particulier lors de ce "Printemps des peuples» qui reste innommé : en témoignent le «Manifeste à l'Europe» de Lamartine ${ }^{10}$, les «États-Unis d'Europe» dont Victor Hugo prophétise l'avènement au Congrès de la paix de 1849, ou encore la «Démocratie européenne» que Victor Considerant appelle de ses vœux ${ }^{11}$. Mais, en

8. Voir la célèbre lithographie de Frédéric Sorrieu, intitulée « République universelle démocratique et sociale. Le Pacte", 1848, Musée Carnavalet.

9. Grand détail de toutes les fédérations et réunion des citoyens et gardes nationales de toute l'étendue du royaume de France, et leur serment de fidélité à la religion, Paris, imp. de C. F. Perlet, s.d., c. 1790, p. 5.

10. Paris, Pagnerre, 1848.

11. Victor Considerant, Représentant du Peuple, L'Apocalypse ou la prochaine rénovation démocratique et sociale de l'Europe [1848], Paris Librairie socialiste phalanstérienne, 1849. 
France, la référence au «Printemps des peuples» reste extrêmement rare dans le vocabulaire politique des contemporains de l'événement.

Les premières traces sont largement postérieures à la période et quasisystématiquement liées au commentaire de la vie politique allemande. Ainsi lorsque Le Gaulois rend compte de l'anniversaire de Bismarck en $1894^{12}$ ou dans un discours de Kurt Eisner en 1918 ${ }^{13}$. L'historien Arthur Chuquet, évoquant en 1905 l'œuvre de Schiller devant les membres de l'Académie des sciences morales et politiques, présente l'un des personnages de l'œuvre du dramaturge allemand comme un annonciateur du "Printemps des peuples ${ }^{14}$. Mais l'expression est absente de l'historiographie de langue française jusqu'à ce que paraisse en 1948 l'ouvrage collectif dirigé par François Fejtö, 1848 dans le monde. Le Printemps des peuples ${ }^{15}$. Les deux volumes de l'ouvrage, qui réunissent une quinzaine de monographies nationales, sont précédés par une forte introduction de leur maitre d'œuvre, polyglotte accompli, ce qui lui permet d'accéder à des sources et à une bibliographie véritablement européennes. Sa connaissance approfondie de l'histoire de la Mitteleuropa l'amène à brosser une large fresque géographique et chronologique de l'avant-1848 ou Vormärz remontant à 1830, voire aux traités de 1815. Si son inspiration marxiste - critique - est aisément repérable, Fejtö accorde une large place aux circulations d'idées en Europe, confronte les idéologues (principalement français et allemands), débat des formes divergentes du nationalisme qui s'épanouissent alors.

Mais jamais il n'explicite l'expression de «Printemps des peuples» qu'il a pourtant choisie comme titre de l'ouvrage et dont on comprend, à lire ses propos, qu'elle n'est pas sans rapport avec la situation de l'Europe en 1948, entrant dans une sorte d' "hiver des peuples» ou d'anti-printemps des peuples. La notion de "Guerre froide» - dont la paternité est attribuée à George Orwell dès $1945^{16}$ - et les nombreuses métaphores saisonnières qui l'accompagnent (glaciation, réchauffement, dégel) sont, vues de l'Ouest, l'antithèse du "Printemps des peuples». En commémorant l'abolition de l'esclavage, on fait de 1948 le centenaire de la liberté, à laquelle est opposé l'asservissement des nations à l'Est de l'Europe. À l'inverse, dans les démocraties populaires naissantes, on célèbre l'avènement de sociétés égalitaires fondées sur les principes marxistes revus et complétés par Lénine et Staline. Évoquant le centenaire du Manifeste du parti communiste, l'historien français Jean Bruhat, membre du Parti communiste, en affirme l'actualité : dénon-

12. Le Gaulois, 2 avril 1894.

13. Le Gaulois, 28 décembre 1918.

14. Arthur Chuquet, "L'ouvre de Schiller", in Séances et travaux de l'Académie des sciences morales et politiques, 65e année, tome 64, 2e semestre, décembre 1905, A. Picard et fils, séance du 6 mai 1905, p. 24. Il s'agit du marquis de Posa, l'un des personnages de Don Carlos (1787).

15. Paris, Minuit, 1948, 2 volumes.

16. George Orwell, 'You and the Atomic Bomb', The Tribune, 19 octobre 1945. Dans cet article, Orwell évoque le cas d'un État qui, détenteur de la bombe atomique, serait dès lors "in a permanent state of 'cold war' with its neighbors". 
çant la "trahison" du gouvernement socialiste français et celle du gouvernement travailliste britannique, il en appelle à la libération de l'Homme par le marxisme, annonciateur d'un nouveau "Printemps des peuples ${ }^{17}$.

En France, les rencontres et publications scientifiques relatives au centenaire de 1848 sont largement centrées sur la Seconde République stricto sensu et n'envisagent souvent la question du «Printemps des peuples» que dans sa relation avec l'évolution de la situation intérieure française - quand la question n'est pas purement et simplement ignorée. C'est aussi bien le cas dans la revue communiste Europe, dirigée par Jean Cassou, que dans Maintenant, un recueil de documents et d'études publié sous la direction de Henry Poulaille ${ }^{18}$. Le même francocentrisme se retrouve dans l'album de la Bibliothèque du centenaire de 1848, agrémenté de textes de Georges Bourgin et de Max Terrier ${ }^{19}$, et dans l'exposition de la Bibliothèque nationale qui consacre toutefois une rubrique aux «révolutions européennes» : mais, tout en reconnaissant que des mouvements insurrectionnels ont précédé la révolution de Février, le catalogue affirme que ceux-ci ont été « déterminés, puis intensifiés par les événements de France ${ }^{20}$. Le Congrès historique du centenaire, qui s'est déroulé à la Sorbonne, comporte une section consacrée à l'histoire politique, divisée en deux sous-sections, dont l'une prend en charge les "pays étrangers à la France »" ${ }^{21}$. Si Pierre Renouvin expose «l'idée d'États-Unis d'Europe pendant la crise de $1848 »^{22}$, on ne peut qu'être frappé par l'absence de l'expression "Printemps des peuples» dans un volume dont le mot-clef - très labroussien - est celui de "crise». Parmi les seize études de cas représentant huit nations, aucun titre ne s'y réfère; en revanche, le mot "social» apparait à quatre reprises et le mot «agraire» une fois. Est-ce à dire que la question des nationalités n'a pas bonne presse auprès des organisateurs du colloque? Que le climat de pré-guerre froide qui se dessine dans l'Europe d'après-guerre, en particulier avec le "coup de Prague» qui vient de se produire, incite à une sorte de prudence sémantique? Ou qu'une lecture marxiste de l'histoire - dominante sans être exclusive - privilégie les infrastructures économicosociales par rapport aux superstructures politico-nationales?

Ce tropisme d'une avant-garde française incarnant ce que Jules Michelet appelait «l'Idée» s'est perpétué jusqu'à nos jours. En 1958, critiquant avec virulence la politique algérienne du régime gaulliste, Roger Garaudy affirme que la France de Massu et des ratissages de la Casbah n'est pas «la France de 1848 qui a donné le signal du "Printemps des peuples" en annonçant

17. Jean Bruhat, Le Centenaire du Manifeste. L'Europe, la France et le mouvement ouvrier en 1848, Paris, Éd. sociales, s.d.

18. "Centenaire de la révolution de 48", Europe, n 26, février 1948; Maintenant, Recueil publié sous la dir. de Henry Poulaille, $n^{\circ} 9$ et 10 , Paris, Grasset, s.d.

19. Bibliothèque du centenaire de 1848, Paris, Éditions Tel, 1948.

20. La Révolution de 1848, Exposition organisée par le Comité national du centenaire, Paris, Bibliothèque nationale, 1948, p. 142.

21. Actes du congrès historique du centenaire de la révolution de 1848, Paris, PUF, 1948.

22. Ibidem, p. 31-46. 
le droit des nations de disposer d'elles-mêmes ${ }^{23}$. Un demi-siècle après le centenaire, les choses n'ont guère évolué, à lire les trois numéros que la Revue d'histoire du XIXe siècle a fait paraître à l'occasion du cent-cinquantenaire de 1848 : sur les 22 articles publiés, 20 ont la France pour cadre ${ }^{24}$. Quant au colloque du cent-cinquantenaire, organisé par la Société d'histoire de la révolution de 1848 et des révolutions du XIX ${ }^{\mathrm{e}}$ siècle, il consacre bien une partie de son programme au printemps des peuples, mais les cas traités le sont sur un registre national (Suisse, Allemagne, Autriche, pays roumains, Royaume-Uni, Portugal) et non comparatif ou transversal, à l'exception de la contribution de Christophe Charle traitant des intellectuels en $1848^{25}$. En revanche, le cent-cinquantenaire a été marqué par la co-production entre la France, l'Italie, la Suisse et l'Allemagne d'une exposition consacrée au «Printemps des peuples ${ }^{26}$. Mais, encore en 2011, dans une contribution au Monde diplomatique intitulée "1848, le printemps des peuples", Alain Garrigou entame son propos par l'affirmation suivante : «En 1848, le printemps commence le 22 février, à Paris $»^{27}$.

\section{UN PRINTEMPS ESSENTIELLEMENT ALLEMAND}

Pour saisir la généalogie du "Printemps des peuples", il est nécessaire de décaler le regard du côté de l'espace germanique. Dès 1789, l'écrivain et pédagogue Joachim Heinrich Campe, auquel l'Assemblée législative accorda le titre de citoyen français en 1792, évoque dans ses Lettres de Paris l'espoir que la Révolution française serait le «Printemps de l'intérêt général des peuples» («der Frühling des allgemeinen Völkerwohls»), inscrivant son propos dans une dimension européenne revendiquée ${ }^{28}$. Depuis les travaux du philologue allemand Otto Ladendorf ${ }^{29}$, on sait que l'écrivain et journaliste Carl Ludwig Börne (1786-1837) a joué un rôle pionnier dans la diffusion de l'expression "printemps des peuples». La trajectoire de ce républicain hessois,

23. Roger Garaudy, «Note sur démocratie et nation", La Pensée. Revue du rationalisme moderne, $\mathrm{n}^{\circ} 80,1958$, p. 22.

24. Francis Démier et Jean-Luc Mayaud [dir.], "Cinquante ans de recherches sur 1848", Revue d'histoire du XIX" siècle, n' ${ }^{\circ}$ 14, 1997; Jean-Claude Caron et Michèle Riot-Sarcey [dir.], «1848. Nouveaux regards ", Revue d'histoire du XIXe siècle, n 15, 1997; Pierre Lenoël et Jean-Jacques Yvorel [dir.], «1848. Un modèle politique à l'épreuve», Revue d'histoire du XIX siècle, n 16, 1998.

25. Christophe Charle, «L'Europe des intellectuels en 1848», in Jean-Luc Mayaud [dir.], 1848. Actes du colloque international du cent-cinquantenaire, Grâne, Créaphis, p. 421-447.

26. Cf. le catalogue Les révolutions de 1848. L'Europe des images. Le printemps des peuples, Paris, Assemblée nationale, janvier 1998. Cette exposition a été organisée en partenariat avec l'association "Torino Citta Capitale Europea», le Musée national suisse de Prangins et le Germanisches Nationalmuseum de Nuremberg. Elle fait miroir au premier volet de l'exposition, «Une République nouvelle», consacrée au seul cas français.

27. Le Monde diplomatique, mai 2011, p. 27.

28. Cité in Jost Hermand (Hrsg.), Von deutscher Republik 1775-1795. Texte radikaler Demokraten, Frankfurt am Main, Insel Verlag, 1968, p. 101-104 et 118-121. Lettre datée du 26 août 1789.

29. Auteur de Historiches Schlagwörterbuch. Ein Versuch, Strassburg/Berlin 1906. 
très impliqué dans le mouvement Jeune-Allemagne, exilé à Paris où il mourut et est enterré, est représentative d'une période où le combat politique pour la «liberté» et, dans le cas allemand, pour «l'unité», entraîne leurs promoteurs sur les routes de l'exil. On peut rapprocher le cas de Börne de ceux de Georg Büchner (mort la même année à Zurich) et de Heinrich Heine, puis des émigrés allemands des années 1840 (Weitling, Marx, Ruge, les époux Herwegh, etc. ${ }^{30}$ L'expression Völkerfrühling est attestée dans Die Waage ( $\mathrm{La}$ Balance), le périodique publié par Börne à Francfort de 1818, au lendemain de la fête de la Wartburg, jusqu'à sa suppression par la censure en $1821^{31}$. Dès l'annonce de la publication du journal, Börne se fait le prophète joyeux du proche avènement du «Printemps des peuples $»^{32}$. Si l'usage de la formule ne dépasse pas le cadre du lectorat bourgeois du périodique, il tend toutefois à être repris par d'autres, notamment dans les années 1830, où il s'implante durablement dans le vocabulaire politique ${ }^{33}$.

Ces années sont marquées par un réveil national dont témoignent en 1832 la fête de Hambach, étape-clef du Vormärz, et, un an plus tard, la "Frankfurter Wachensturm», ou attaque de la garde de Francfort-sur-leMain, une tentative révolutionnaire radicale qui échoua. Le fait que cette dernière se soit déroulée au mois d'avril et la fête de la Hambach au mois de mai accentue la métaphore printanière de ce Risorgimento allemand. Par certains aspects, ce réveil culturel en Europe n'est pas sans parenté avec la Renaissance, considérée dès le milieu du XVI e siècle comme le réveil d'hommes endormis qui, «après l'aspre saison de l'hiver reprennent leur vigueur à la chaleur du Soleil et sont consolés de la douceur du printemps " ${ }^{34}$. Si la figure poétique d'un printemps régénérateur irrigue constamment la poésie européenne, elle est particulièrement présente dans le romantisme allemand et les noms de Goethe - voir son poème de 1771, Maifest, hymne au renouveau de la nature -, de Kleist, "le chantre du printemps »35, ou encore Rückert célébrant en 1844 le Printemps d'amour (Liebesfrühling en allemand) y sont

30. Pour des éléments biographiques sur plusieurs d'entre eux, voir Karl Marx et Friedrich Engels, Les Grands hommes de l'exil, texte inédit en français, éditions établie et préfacée par Sylvie Aprile, Marseille, Agone, 2015.

31. Die Wage (sic). Eine Zeitschrift für Bürgerleben, Wissenschaft und Kunft, herausgegeben von Dr Ludwig Börne, Frankfurt-am-Main, 1818. Heinrich Heine, Ludwig Börne. A Memorial, translated with commentary and an introduction by Jeffrey L. Sammons, Rochester, Camden House, 2006, not. p. XVI.

32. Otto Ladendorf, op. cit., "Völkerfrühling», consulté en ligne sur http://www.textlog.de/ schlagworte-voelkerfruehling.html

33. Rudolf Jaworski, "Völkerfrühling 1848», in Dieter Langewiesche (Hrsg.), Demokratiebewegung und Revolution 1847 bis 1849. Internationale Aspekte und europä̈sche Verbindungen, Berlin, Heidelberg, Springer Verlag, 1998, p. 36-51; Christian Pletzing, Vom Völkerfrühling zum nationalen Konflikt. Deutscher und polnischer Nationalismus in Ost- und Westpreussen 1830-1871, Wiesbaden, Harrasowitz Verlag, 2003.

34. Pierre Belon du Mans, Les Observations de plusieurs singularitez et choses memorables, 1553, cité par Arlette Jouanna, "La notion de Renaissance : réflexions sur un paradoxe historiographique", Revue d'histoire moderne et contemporaine, 2002/5, p. 4.

35. François-Xavier de Feller, Biographie universelle ou Dictionnaire historique, tome I, Besançon, Outh.-Chalandre fils, et Paris, Méquignon junior et J. Leroux, Gaume frères, 1844, p. 174. 
étroitement associés. Il serait hasardeux de donner une interprétation politique à cette production poétique, mais elle a pu contribuer à enraciner l'idée d'une régénération politique à la fois naturelle et inévitable, comme l'est le rythme des saisons.

Craint ou espéré, le printemps des peuples résume en une formule parlante les enjeux du combat politique qui oppose "libéraux" et "conservateurs" sur le devenir de l'Allemagne. C'est ainsi qu'un contemporain de Börne, Jordanus Brunow, pseudonyme d'August Gatyh, publie en 1831 une brochure intitulée Le Printemps des peuples et ses prophètes. Salut printanier aux orateurs de l'Allemagne ${ }^{36}$, qui sonne comme une vibrante apologie du combat pour la liberté, exemple de la Révolution française à l'appui. Le même caractère prophétique se retrouve dans deux romans de l'écrivain et journaliste Theodor Mundt, lui aussi proche du mouvement Jeune Allemagne ${ }^{37}$. La référence à la France comme modèle politique - qu'il soit admiré ou rejeté - reste constante jusqu'aux révolutions de 1848. Börne lui-même s'en fait le relais dans la version française de La Balance qu'il publie à Paris en $1836^{38}$. La répartition des rôles qu'il propose - la France sape l'ordre ancien, l'Allemagne construit le nouveau - intéresse moins par les stéréotypes remis au goût du jour que par la contradiction qui affleure dans son propos entre l'idée de «nation allemande» et celle de "peuples germaniques» ou parfois "les peuples de langue allemande " ${ }^{39}$. Il s'interroge pourtant sur ce qu'est la "patrie» pour les Allemands, entre morcellement politique et mythe impérial ${ }^{40}$. À lire Börne, on saisit que le «Printemps des peuples» qu'il envisage est composé d'éléments qui s'articulent difficilement : revendication de la liberté, attachement à la double patrie (Heimat/Vaterland), construction d'un roman national où la mythologie percute l'histoire.

Toutes choses que la France semble avoir dépassées, malgré (ou grâce à?) la défaite de 1815 : l'enracinement du sentiment national et patriotique est ressenti comme un acquis, tout comme la conquête de la liberté. Un journal allemand résume le tricolore à la française par trois mots : "Constitution, Freiheit, Völkerfrühling» (Constitution, liberté, Printemps des peuples) mais ce ne sont que des mots, grince l'auteur ${ }^{41}$. On sait également le regard critique, volontiers ironique, mais parfois admiratif que Heine l'exilé a porté sur le règne de Louis-Philippe ${ }^{42}$. Mais cette verve s'exerce aussi à l'encontre de

36. Der Völkerfrühling und seine Verkünder. Frühlingsgruss an Deutschlands Redner, Nuremberg, Hoffmann et Campe, 1831.

37. Moderne Lebenswirren. Briefe und Zeitabenteuer eines Salzschreibers. Reichenbach, Leipzig 1834 ; Madonna. Unterhaltungen mit einer Heiligen. Reichenbach, Leipzig 1835.

38. La Balance. Revue allemande et française publiée par L. Boerne, volume 1, $1^{\text {re }}$ livraison, janvier, Paris, au bureau de la Balance, 1836.

39. Ludwig Börne, Lettres écrites de Paris pendant les années 1830 et 1831, Paris, Paulin, 1832, p. 94.

40. "Béranger et Uhland", La Balance, 1836, p. 34.

41. "Bericht über deutsche Poeten aus dem Jahre 1841 ", Blätter für literarische Unterhaltung, $\mathrm{n}^{\circ} 301,28$ octobre 1842

42. De la France, Paris, E. Renduel, 1833; Lutèce. Lettres sur la vie politique, artistique et sociale de la France, Paris, M. Lévy frères, 1855. 
ses compatriotes, comme en témoigne un poème de 1842, Atta Troll. Heine y caricature les communistes et autres collectivistes allemands, incarnés par l'ours révolutionnaire qu'est Atta Troll. Il y ferraille aussi contre les libéraux allemands proches de Ludwig Börne, animalisés comme dans la Vie privée et publique des animaux de Granville : "Quel est donc ce bruissement qui fait trembler le monde? Ce sont les gigantesques hannetons du printemps du peuple qui s'avancent, saisis d'une fureur de sauvages ${ }^{43}$.

Quant au poète Ferdinand Freiligrath, qui écrit dans la Neue Rheinische Zeitung et est alors proche de Marx, il voit en 1848 «l'année du Printemps des peuples ${ }^{44}$. Pour autant, la formule ne comporte aucune dimension sociale. La pluralité des peuples les désigne bien comme des entités nationales, là où la singularité $d u$ peuple en fait un acteur de la transformation sociale. Les "peuples" qui font ce printemps de 1848 sont pour l'essentiel composés de classes moyennes, ces «bourgeoisies» économiques et intellectuelles qui, dans un inquiétant climat de crise socioéconomique, n'entendent guère "populariser» leur action unitaire. Le sens de l'expression doit être relié à l'émergence d'un sentiment national allemand concomitant à la «bataille des nations" (Völkerschlacht, Leipzig, octobre 1813) et à la chute de l'Empire napoléonien en deux temps (1814-1815). Le mot Volk, traduit en français à la fois par peuple et par nation, participe d'une ambiguïté durable quant à la perception de la notion de "Printemps des peuples». Car il ne s'agit pas tant, alors, de célébrer l'émancipation des peuples européens que celui des différentes composantes de l'ex-Saint Empire romain germanique, dissous par Napoléon $1^{\text {er }}$. La fragile reconstruction à laquelle ce dernier avait procédé ayant échoué, le contexte semble favorable à une unification dont l'extension territoriale reste mal définie, mais est susceptible d'englober une large partie des "peuples» allemands sur la base d'un libéralisme adossé aux principes de la monarchie constitutionnelle.

On est frappé par l'importance des revendications culturelles et en particulier linguistiques qui s'expriment en 1848 : le "Printemps des peuples» affirme pour la première fois à l'échelle européenne le droit de s'exprimer, publier, enseigner, prier dans une langue nationale constituant le dénominateur commun d'individus que tout sépare par ailleurs. La langue devient un vecteur essentiel de l'affirmation identitaire, en particulier chez les nations slaves. Loin de souscrire à l'image d'un peuple un et unique à vocation internationaliste - comme Marx et Engels en avaient posé l'hypothèse dans le Manifeste du Parti communiste -, l'expression de "Printemps des peuples»

43. Cité par Louis Reynaud, "La source française d'Atta Troll», Revue germanique, $10^{e}$ année, $\mathrm{n}^{\circ}$ 1, 1914, p. 154. Texte allemand: "Welch ein Sumsen, welterschütternd! Das sind ja des Völkerfrühlings Kolossale Maienkäfer, Von Berserkerwuth ergriffen!». Il existait une "Ligue des hannetons de mai" publiant son journal, Der Maikäfer: eine Zeitschrift für Nicht-Philister (Le Hanneton de mai : un journal pour les non-Philistins), fondée en 1840 par Gottfried et Johanna Kinkel. Cette ligue de poètes, parmi lesquels Ferdinand Freiligrath et Georg Weerth, s'inscrivait dans une sensibilité socialiste. Cf. Karl Marx et Friedrich Engels, Les Grands hommes de l'exil, op. cit.

44. Le XIXe siècle, 10 mars 1904. 
renvoie à l'idée de communautés nationales regroupées derrière un drapeau. Cette pluralité traduit le sentiment que le printemps 1848 correspond à la fin de l'Europe de la Sainte-Alliance ou de l'Europe des rois, qui laisserait la place à l'Europe des peuples ou à l'Europe des nations, principe intimement lié à l'affirmation du libéralisme. On peut rappeler l'initiative du Vénitien Niccolo Tommaseo, fondateur de la "Società della Fratellanza de' Popoli " et du journal du même nom qui, dans Venise assiégée, perpétue les idéaux du Printemps des peuples. Ce faisant, Tommaseo annonce cette «fraternité européenne» célébrée par Victor Hugo lors du Congrès de la paix de Paris en août 1849, alors que Venise capitule sous les bombes autrichiennes. Du reste, l'espace européen envisagé par Tommaseo se contracte et se recentre en direction des peuples adriatiques, balkaniques et hongrois ${ }^{45}$.

Les «années 1848" représentent un tournant dans la perception et la réception du "Printemps des peuples». Car l'échec de l'unité allemande qui ressort de ces années se traduit par un double mouvement d'enracinement populaire et de récupération impérialiste de la notion. Celle-ci est en effet revendiquée par le nationalisme prussien, qui en fait la matrice du Reich à construire. En 1865, l'historien allemand Heinrich von Sybel, évoquant la chute de Napoléon un demi-siècle plus tôt, analyse l'année 1815 en pays rhénan comme "un incomparable Printemps des peuples " ${ }^{46}$. Objet d'une véritable captation politique, l'expression est reprise à son compte vingt ans plus tard par le chancelier Bismarck qui fixe le début du Printemps des peuples à l'année 1866, sa continuation en 1870 et son aboutissement avec la colonisation menée au nom de la Weltpoliti $k^{47}$. Dans un grand discours prononcé devant le Reichstag en mars 1885 , celui qui fut un farouche adversaire de l'Unité allemande voulue par le Parlement de Francfort déclare : «Je me représentais comme un Printemps des peuples ces événements successifs : la reprise des anciennes frontières, la convocation du Reichstag allemand représentant l'unité nationale et le rétablissement de l'empire "48, concluant : «Le Printemps des peuples, c'est moi qui l'ai créé en créant l'Unité allemande $»^{49}$. On assiste donc, des années 1860 aux années 1890, à une véritable captation de l'idée de Printemps des peuples par les partis nationalistes qui s'approprient un concept permettant de rejeter au second plan les divisions internes apparues en particulier au moment du Kulturkampfou dans le combat entrepris par Bismarck contre la gauche socialiste. Le point culminant est atteint en 1911 dans l'ouvrage d'Otto-Richard Tannenberg, Le Rêve allemand, qui

45. Dominique Kirchner Reill, Nationalists who Feared the Nation. Adriatic Multi-Nationalism in Habsburg Dalmatia, Trieste, and Venice, Stanford, Stanford University Press, 2012; Giordano Altarozzi et Cornel Sigmirean [dir.], Il Risorgimento italiano e i movimenti nazionali in Europa. Dal modello italiano alla realtà dell'Europa centro-orientale, Roma, Edizioni Nuova Cultura, 2013.

46. Cité par Julien Rovere, "La rive gauche du Rhin. I. La résistance à la conquête (1815-1848) ", Revue des deux mondes, tome XLI, $1^{\mathrm{er}}$ septembre 1917, p. 515.

47. Discours du 14 mars, cité dans Le Figaro, 18 mars 1885.

48. Journal des Débats, 15 mars 1885.

49. La Croix, 8 avril 1885. 
en appelle à un "second printemps des peuples" pour achever la germanisation des provinces orientales de l'Europe ${ }^{50}$. Toutefois, la force évocatrice de la notion, en particulier pour les peuples de l'Empire austro-hongrois luttant pour leur indépendance, amène un marxiste patenté comme Karl Kautsky à prophétiser en 1902 l'avènement d'un "nouveau, un radieux Printemps des peuples» dont le centre sera la Russie ${ }^{51}$. Encore dans l'entre-deux-guerres, la notion de «Völkerfrühling» continue d'irriguer le discours politique allemand, Gustav Stresemann le mobilisant lors de l'admission de l'Allemagne à la SDN en $1926^{52}$.

\section{RÉACTUALISATIONS}

De nombreux chrononymes ont été sollicités pour désigner des événements postérieurs ayant une parenté apparente avec le modèle originel. Ces réactualisations illustrent moins la validité de la comparaison entre passé et présent que la force de l'imaginaire et l'incapacité à forger des néo-chrononymes. La notion de "Trois glorieuses" a été l'objet d'un détournement bien connu pour désigner les années de croissance économique et démographique ayant suivi la Seconde Guerre mondiale en France - les "Trente Glorieuses", expression popularisée par le livre éponyme de Jean Fourastié paru en 1979. Claude Blanckaert rappelle que l'anthropologue Georges Hervé qualifia en 1909 de «Trois Glorieuses» la création de la Société d'anthropologie de Paris, la reconnaissance officielle de la préhistoire par l'Académie des sciences et la publication de L'Origine des espèces de Charles Darwin ${ }^{53}$. Mais on évoque aussi les Trois Glorieuses des 9, 10 et 11 décembre 1943, au cours desquelles la centrale d'Eysses (Villeneuve-sur-Lot) a été l'objet d'une insurrection visant à empêcher le transfert des internés administratifs en zone nord ${ }^{54}$. Plus récemment, la révolte syndicaliste des 13, 14 et 15 août 1963 qui aboutit au renversement du président congolais Fulbert Youlou a été qualifiée de Trois Glorieuses : un collège de Pointe-Noire en porte le nom, comme le rappelle Alain Mabanckou ${ }^{55}$. L'expression a essaimé au Togo pour désigner trois journées de 1974 marquantes dans l'histoire du pays et, de même, trois datesclefs dans la transformation du Dahomey en Bénin : soit autant de traces du maintien d'une influence culturelle française post-coloniale. On citera égale-

50. Otto-Richard Tannenberg, Le Rêve allemand. La plus grande Allemagne. L'ouvre du XXe siècle [1911], Lausanne-Paris, Payot, 1916, p. 209.

51. Karl Kautsky, «Les Slaves et la révolution ", L'Iskra, journal révolutionnaire social-démocrate russe, $\mathrm{n}^{\circ} 18,10$ mars 1902 .

52. Le Journal des Débats, 7 septembre 1926.

53. Claude Blanckaert, "Les "trois glorieuses de 1859" et la genèse du concept de races historiques", Bulletins et mémoires de la Société d'anthropologie de Paris, vol. 22, n 1-2, 2010, p. 3-16.

54. Voir la contribution de Corinne Jaladieu sur https://criminocorpus.org/fr/musee/eysses/ complements/les-trois-glorieuses/

55. Alain Mabanckou, Lumières de Pointe-Noire, Paris, Seuil, 2013. 
ment les dérivés plus ou moins consacrés comme les "Trente piteuses», titre d'un ouvrage de Nicolas Baverez qui se réfere explicitement à Fourastiée ${ }^{56}$. Et il n'est pas jusqu'à Laurent Fabius qui, ouvrant le colloque de trois journées consacré au cent-cinquantenaire de 1848, ne souhaite le meilleur aux participants à ces «trois studieuses ${ }^{57}$...

Il existe de même, en dehors de la Commune de Paris, d'autres «Semaines sanglantes». La plus célèbre est celle qui se déroule dans les rues de Berlin en janvier 1919, lors de l'affrontement entre groupes spartakistes et forces du Conseil des commissaires du peuple qui a succédé à Guillaume II après son abdication en novembre 1918. Les événements qui, au même moment, ensanglantent les rues de Buenos-Aires à la suite de violentes grèves sont également référencés comme "Semaine sanglante». Le sont aussi les violences antisémites exercées en juin 1933 par les SA contre des juifs berlinois du quartier de Köpenick qualifiées de "Köpenicker Blutwoche " ${ }^{58}$. Mais les usages journalistiques de l'expression sont multiples et il n'est guère de violences urbaines, quels qu'en soient la nature et l'objet, qui ne soient qualifiées de «semaine sanglante» (règlements de compte en série à Marseille, suite d'attentats et d'embuscades en Algérie, fusillades à répétition aux États-Unis, affrontements entre milices en Lybie, affrontements inter-ethniques au Nigéria, guerre en Ukraine, violences intra-communautaires en Israël), jusqu'aux événements survenus à Paris en janvier 201559. Là aussi il existe des dérivés liés notamment à la restriction de la notion à un seul jour de la semaine, dont le plus célèbre exemple est le «Bloody Sunday» irlandais du 30 janvier 1972.

Moins nombreux, les réemplois de la notion de "Printemps des peuples» sont liés à l'émergence, plus ou moins soudaine et générale, de mouvements promouvant à la fois le principe de liberté et celui de nationalité. C'est ainsi que les révolutions de 1848 ont joué un rôle fondamental dans l'émergence d'un sentiment national juif, aux origines du sionisme. L'ouvrage que Moses Hess publie en 1862, Rome et Jérusalem. La dernière question nationale, devait initialement s'intituler Renaissance d'Israël ou Printemps des peuples ${ }^{60}$. Le «Printemps des peuples» est proche de cet «éveil des nationalités» auquel Georges Weill a consacré une solide étude ${ }^{61}$. L'époque de cette publication (1930) correspond à la fin d'un autre Printemps des peuples qui avait émergé dans les années vingt et qui succomba devant la montée des totalitarismes sur fond de crise économique. La comparaison avec 1848 opère d'autant mieux que ce sont de nouveau les nationalités relevant de l'ex-Empire aus-

56. Paris, Flammarion, 1998.

57. Laurent Fabius, «Ouverture», in Jean-Luc Mayaud [dir.], 1848, op. cit., p. 9.

58. http://www.museumsportal-berlin.de/fr/musees/gedenkstatte-kopenicker-blutwochejuni-1933.

59. Hubert Montagner, «Semaine sanglante de janvier 2015. Pour un vrai débat de société», Journal du droit des jeunes, $\mathrm{n}^{\circ} 341,2015 / 1$, p. 35-38.

60. Robert Southard, "Hess, Moses», in Christopher John Murray (ed.), Encyclopedia of the Romantic Era, tome I, New York-Londres, Fitzroy Deaborn, 2004, p. 502.

61. Georges Weill, L'Eveil des nationalités et le mouvement libéral 1815-1848, Paris, PUF, 1930. 
tro-hongrois (tchèque, hongroise, roumaine, polonaise, slaves du Sud) qui apparaissent à la pointe de ce combat nationalitaire et libéral. En 1948, le centenaire des révolutions de 1848 a célébré non sans hésitations ni arrièrepensées un possible nouveau Printemps des peuples. Mais dans le climat grandissant de Guerre froide, marqué par la division de l'Allemagne et de l'Europe dans son ensemble, et plus ponctuellement par le "coup de Prague", la chose semblait déjà relever de l'anachronisme. Mais vingt ans plus tard, les mouvements insurrectionnels tchèques ont été popularisés comme le "printemps de Prague», un chrononyme qui recouvre en fait plus d'un semestre d'histoire tchécoslovaque, de son début en janvier 1968 à son écrasement au mois de juillet suivant : lui donnant une dimension nationale, François Fejtö préfere parler de Printemps tchécoslovaque ${ }^{62}$.

La chute du mur de Berlin et du rideau de fer en 1989 a entraîné une brève résurgence de l'expression. Michaël Jeismann, historien et journaliste au Frankfurter Allgemeine Zeitung, évoque ainsi «les espoirs du bref Printemps des peuples des années 1989 et $1990 »^{63}$. Toutefois, dans un souci de respect de la temporalité saisonnière, René Girault, dans un célèbre article publié dans Le Monde le 4 janvier 1990, préfère évoquer «l'automne des peuples" pour qualifier les effondrements successifs des régimes communistes en Europe centrale et orientale ${ }^{64}$. Girault se réjouit que «la parenthèse gigantesque de révolutions dévoyées prenne fin» et que les Européens aient exprimé « une magnifique volonté de reprendre le cours normal de leur histoire, qui est l'histoire d'une Europe libre et démocratique». Sa lecture de l'événement exclut implicitement la question sociale et la question des nationalismes, dont le brutal réveil a amené certains observateurs des guerres de l'ex-Yougoslavie à parler d' "hiver des peuples " ${ }^{65}$. Le risque d'un glissement du sentiment national vers la résurgence d'un nationalisme exacerbé est apparu comme un danger potentiel - finalement maitrisé.

Rares sont les chrononymes qui accordent une place centrale à un acteur collectif. Mais la mise en exergue des "peuples» de ce printemps 1848 contribue-t-elle à la politisation de l'événement ou à sa récupération mémorielle ${ }^{66}$ ? Comme on l'a vu, le Printemps des peuples existe avant 1848 et se perpétue après, essentiellement dans l'Europe germanique. De Börne à Bismarck, il passe d'une lecture libérale et démocratique à une lecture nationaliste et

62. Bruxelles, Complexe, 1999.

63. Michaël Jeismann, "Nationalisme et identité politique en Allemagne», Bulletin de la Société d'histoire moderne et contemporaine, $\mathrm{n}^{\circ} 1-2,1996, \mathrm{p} .39$.

64. René Girault, «Lautomne des peuples», repris dans Le Monde, 13 octobre 2009.

65. Stéphane Pierre-Caps, La Multination. L'avenir des minorités en Europe centrale et orientale, Paris, Odile Jacob, 1995, p. 139.

66. Paul Bacot, Laurent Douzou et Jean-Paul Honoré, "Chrononymes. La politisation du temps", Mots. Les langages du politique, n 87, 2008, mis en ligne le 21 juillet 2010. 
impérialiste; d'une approche faisant de la réforme et, si nécessaire, de la révolution la clef de l'unité allemande, à une approche célébrant l'armée et la guerre comme moteurs de cette unité. Cela explique pourquoi l'historiographie allemande précise parfois : le "Printemps des peuples de 1848", ce qui, côté français, apparaîtrait redondant ${ }^{67}$. Le contraste n'est que plus net entre une historiographie française encore réticente à incorporer le «Printemps des peuples» dans une dimension réellement européenne à sa grille de lecture de 1848, une démarche entraînant un nécessaire décentrement du regard comme on a pu le faire pour $1830^{68}$. Mais ce qui est vrai de l'historiographie française se vérifie ailleurs : comme Axel Körner l'affirme, le fossé demeure large entre la dimension européenne de l'idéologie quarante-huitarde et la nationalisation persistante de la mémoire de $1848^{69}$.

Quant à l'opération de captation rhétorique et historiographique des révolutions du monde arabe, résumées par la formule "printemps arabe", elle établit un parallèle explicite, mais non démontré avec le Printemps des peuples ${ }^{70}$. Ce qui se voulait, chez certains, un hommage aux révolutionnaires arabes ne faisait en réalité que traduire la persistance de schèmes européocentrés, projetés sur un ailleurs dont l'histoire, le langage, les particularismes culturels étaient impensés ${ }^{71}$. En réaction, des formulations parallèles sont apparues : "printemps arabes», "printemps des peuples arabes», "révolutions arabes ", insistant généralement sur la pluralité et la diversité d'un mouvement hétérogène. Les chrononymes n'ayant au fond qu'une portée symbolique, on pourrait relativiser la critique. Mais tout historien des révolutions sait combien le poids des symboles est déterminant dans leur dénomination. Raison de plus pour que, comme l'affirmait un célèbre slogan de mai 68, l'imagination soit au pouvoir.

Jean-Claude Caron est professeur à l'Université Blaise Pascal-Clermont-Ferrand et membre de l'Institut Universitaire de France

67. Rudolf Jaworski, op. cit.

68. Sylvie Aprile, Jean-Claude Caron et Emmanuel Fureix [dir.], La Liberté guidant les peuples. Les révolutions de 1830 en Europe, Seyssel, Champ Vallon, 2013.

69. Axel Körner, 1848. A European Revolution? International Ideas and National Memories of 1848, London, Palgrave Macmillan, 2002.

70. Pour une analyse des débats autour de la question, voir Guillaume Mazeau, «La ronde des révolutions", en ligne sur laviedesidées.fr, http://www.laviedesidees.fr/IMG/pdf/20130416_ronde_ des_revolutions.pdf

71. Jean-Claude Caron, "Trois Glorieuses", "Printemps des peuples", "Semaine sanglante" : quand la métaphore corsète la révolution (France, XIX ${ }^{\mathrm{e}}$ siècle)", in Habib Belaïd, Kmar Bendana, Sihem Kchaou [dir.], Thawra(t). Approche comparée des révoltes et révolutions (XIXe-XXIe siècle), Tunis, Université de la Manouba - Institut Supérieur d'Histoire de la Tunisie Contemporaine, 2014, p. $15-25$. 